\title{
LTE model atmospheres with new opacities
}

\section{Methods and general properties ${ }^{\star}$}

\author{
N. T. Behara and C. S. Jeffery
}

Armagh Observatory, College Hill, Armagh BT61 9DG, Northern Ireland

Received 3 August 2005 / Accepted 13 January 2006

\begin{abstract}
We describe an opacity sampled version of the LTE model atmosphere code STERNE with revised continuous opacities. In particular, we examine the results of replacing the existing treatment of the continuous opacities which followed the method of Kurucz (1970, SAOSR, No. 309) and Peach (1970, MNRAS, 73, 1), with photoionization cross-sections from the Opacity Project and the IRON Project, and the effects of substituting an opacity distribution function method for treating the line opacities with an opacity sampling method. In studying the application of these new models to atmospheres with hydrogen-rich and extreme chemical compositions, we find the new opacity treatment increases the temperature in the line forming region by up to $3000 \mathrm{~K}$ for extremely hydrogen-poor stars and up to $1250 \mathrm{~K}$ for hydrogen-rich stars with $T_{\text {eff }}=30000 \mathrm{~K}$ and $\log g=3.00$. This translates into systematically cooler effective temperature measurements for these types of stars.
\end{abstract}

Key words. atomic data - methods: numerical - stars: atmospheres - stars: chemically peculiar - stars: fundamental parameters

\section{Introduction}

The general problem of radiative transfer in stellar atmospheres in the limiting approximation of a plane-parallel semi-infinite stellar atmosphere in hydrostatic and local thermodynamic equilibrium has been treated extensively. While it is now common to consider departures from this approximation, there remain problems of considerable interest which need to be explored within it. The specific problem addressed here is that in which hydrogen is absent; this has important consequences for the way in which opacity is treated within a model atmosphere. Normally hydrogen is the dominant source of opacity in a stellar atmosphere; this has been an implicit assumption in the majority of treatments (e.g. ATLAS, Kurucz 1970, 1993). The absence of hydrogen, as encountered for example in extreme helium stars and R Coronae Borealis stars, places an increased onus on the accuracy and completeness of other opacity sources.

Very few codes for the calculation of stellar atmospheres have been written specifically to address this problem. The two principal examples are STERNE (Schönberner \& Wolf 1974) and MARCS (Gustafsson et al. 1975). To compensate for the low hydrogen opacity, the original version of STERNE considered in detail more sources of continuous opacity than other contemporary codes, particularly carbon and nitrogen. However, in the thirty years since STERNE was first written, theoretical work has provided superior calculations of continuous

* Appendix B is only available in electronic form at http://wWw.edpsciences.org opacities both for carbon, nitrogen and also for many other ions (Berrington 1995). The contribution of absorption lines to the overall opacity has also seen major advances. Most model atmosphere codes now include a treatment of line opacity, either by the use of opacity distribution functions (ODF) (ATLAS6, Kurucz 1970), opacity sampling (Peytremann 1974; ATLAS12, Kurucz 1996; MARCS, Asplund et al. 1997) or complete line synthesis (Shulyak 2004). While the ODF approach has since been implemented in STERNE (Heber et al. 1984), the available ODFs use only $\approx 10^{5}$ lines and are restricted in composition.

In addition to improvements in opacity, many modern studies of stellar atmospheres also consider departures from local thermodynamic equilibrium (non-LTE) (e.g. TLUSTY, Hubeny \& Lanz 1995; PRO2, Werner 1986; PHOENIX, Hauschildt 1992). While departures from LTE are evident in the profiles of strong lines in stars of spectral types A and B, it remains the case that these are less important for the structure of the atmosphere as a whole than is a complete treatment of the continuous and line opacities (Anderson \& Grigsby 1991; Przybilla et al. 2005). Moreover, since LTE models can be computed relatively cheaply compared with full non-LTE calculations, they are very important for exploring large volumes of parameter space.

The model atmosphere code STERNE ${ }^{1}$ assumes the stellar atmosphere can be approximated by a plane-parallel geometry and that it is in local thermodynamic, radiative and hydrostatic

\footnotetext{
1 The name STERNE was attached to the code when it was submitted to the CCP7 program library in the mid 1980's (Jeffery 1989).
} 
equilibrium (LTE). It had evolved in stages from a code developed to study planetary nebula central stars (Böhm \& Deinzer 1965). Hunger \& Van Blerkom (1967) subsequently applied it to the case of a pure helium atmosphere. Additional boundfree opacities were introduced by Schönberner \& Wolf (1974), and Heber \& Schönberner (1981) incorporated line blocking from $52 \mathrm{He}$ I lines and $400 \mathrm{UV}$ metal lines via the synthetic spectrum approach. An ODF approach to treat line opacities was introduced by Heber et al. (1984) for hydrogen-rich compositions. Möller (1990) computed an ODF for a hydrogendeficient mixture from the Kurucz \& Peytremann (1975) list of 265000 lines.

Models computed with this H-deficient ODF were used in analyses of extreme helium stars by Jeffery \& Heber (1992) and in more than ten subsequent papers up to and including Pandey et al. (2005). Descriptions of improvements to the code since 1984 were given by Jeffery \& Heber (1994) and Jeffery et al. (2001). An extensive grid of model atmospheres computed with the latest version of STERNE is available online at wWw . arm. ac . uk/ csj/models/Grid. html.

As a consequence of advances in the calculation of theoretical photoionization cross-sections and the availability of more extensive data for atomic absorption lines, these models are now seriously out of date. In view of the continuing importance of the models computed with LTE approximation, a major revision of STERNE has been long overdue. This revision has been completed and is presented in this paper. The new opacities and methods are described in detail, while the principal consequences for the models are discussed. A subsequent paper will apply these models to the specific case of the extreme helium stars.

\section{Opacity}

We describe how the continuous and line opacity data has been revised in STERNE, using photoionization data from the Opacity Project (OP, Seaton et al. 1994) and the IRON Project (IP, Hummer et al. 1993), and compilations of oscillator strengths and damping constants from Kurucz (1995). For completeness, the current treatment of the scattering coefficient is described in the appendix.

\subsection{Bound-free absorption: opacity project cross-sections}

The absorption coefficient due to photoionization, or boundfree continuous opacity, represents the energy required to ionize an electron from a given bound state. Up to 2003 the bound-free opacities for $\mathrm{HI}, \mathrm{H}^{-}, \mathrm{He}$ II, $\mathrm{He}^{-}, \mathrm{C}^{-}, \mathrm{O}$ I, $\mathrm{Mg}$ I, Mg II, AlI, Si I, Si II, and Ca II were treated in STERNE as in ATLAS (Kurucz 1970). These were supplemented by additional tables for carbon and nitrogen, C I, C II, C III, N I, N II, and N III (Peach 1970). The Kurucz and Peach bound-free absorption coefficients were calculated using a general formula for atomic photoionization cross-sections together with estimated correction terms to the hydrogenic coefficient, calculated using exact Gaunt factors. The He I terms with quantum number $n=3$ and $n \geq 4$ were treated as described by
Hunger \& Van Blerkom (1967). Koester (1985) introduced new cross-sections for the $n=1$ and $n=2$ terms. For the transitions from the $1^{1} \mathrm{~S}, 2{ }^{1} \mathrm{~S}$ and $2{ }^{3} \mathrm{~S}$ terms the cross-sections were replaced with calculations from Stewart (1978a, 1978b, 1979). Calculations for the $2{ }^{1} \mathrm{P}$ and $2{ }^{3} \mathrm{P}$ terms were performed using the quantum defect method described by Burgess \& Seaton (1960) and Peach (1967). Of all these bound-free opacities, only the treatments for $\mathrm{H}^{-}, \mathrm{He}^{-}$and $\mathrm{C}^{-}$have been retained.

For photon energies below the ionization threshold, the cross-section is zero; for energies above the threshold, the cross-section is non-zero, decaying approximately as $v^{-3}$ for increasing $v$. During the Opacity Project's $(1995,1997)$ comprehensive re-derivation of photoionization and photoexcitation cross-sections, it was discovered that photons with energies below the threshold could ionize the ion due to resonances with bound-bound transitions - known as photoexcitation of the core or PEC resonances, resulting in large deviations from the smooth hydrogenic functions. Any solution of the non-grey radiative transfer problem in stellar atmospheres should take these contributions to the opacity into consideration, as well as including the substantial improvement to the continuous opacity in well-behaved domains provided by the Opacity Project and other modern studies.

The Opacity Project computed monochromatic photoionization cross-sections $\alpha_{i j k}(v)$ for each of the lowest-lying electron states $i$ in $\mathrm{H}, \mathrm{He}, \mathrm{Li}, \mathrm{Be}, \mathrm{B}, \mathrm{C}, \mathrm{N}, \mathrm{O}, \mathrm{F}, \mathrm{Ne}, \mathrm{Na}, \mathrm{Mg}, \mathrm{Al}$, $\mathrm{Si}, \mathrm{S}, \mathrm{Ar}, \mathrm{Ca}$ and $\mathrm{Fe}$ where $j$ denotes the ion species of the element $k$. These have been incorporated, with the exception of the iron cross-sections, by computing the average contribution of each electron state within each wavelength interval $2 \Delta$ in the model atmosphere calculation

$\bar{\alpha}_{i j k}(\lambda)=\frac{\int_{\lambda-\Delta}^{\lambda+\Delta} \alpha_{i j k}\left(\lambda^{\prime}\right) \mathrm{d} \lambda^{\prime}}{\int_{\lambda-\Delta}^{\lambda+\Delta} \mathrm{d} \lambda^{\prime}}$.

These averages are computed on the model atmosphere wavelength grid from the tabulated cross-sections at program initialization. The total cross-section per atom is obtained by summing over electron state populations $\xi_{i j k}\left(\sum_{i} \xi_{i j k}=1\right)$ and ion abundances $\beta_{j k}$

$\alpha_{\mathrm{bf}}(\lambda)=\sum_{i j k} \xi_{i j k} \beta_{j k} \bar{\alpha}_{i j k}(\lambda)$

and transformed to give the total continuous absorption coefficient per gram $\kappa$ from

$\kappa_{\mathrm{bf}}(\lambda)=\frac{N_{\mathrm{A}}}{\mu_{\mathrm{I}}} \alpha_{\mathrm{bf}}(\lambda)$

The way in which the OP data is included in the model atmosphere code is sufficiently general that any combination of ions may be selected, once the necessary cross-sections have been downloaded from TOPbase (Cunto et al. 1993). The ions currently included, together with the number of states treated for each ion $\left(n_{i j}\right)$, are shown in Table 1.

Because OP cross-sections were calculated from theoretical model atoms in which the electron energy levels were up to $10 \%$ different from observed values, photoionization edges do 
Table 1. Photoionization data included.

\begin{tabular}{lll}
\hline \multicolumn{3}{l}{ Opacity project } \\
$k$ & $j$ & $n_{i j}$ \\
\hline $\mathrm{H}$ & I & 55 \\
$\mathrm{He}$ & I, II & 53,55 \\
$\mathrm{Li}$ & I, II, III & $25,53,55$ \\
$\mathrm{Be}$ & I, II, III, IV & $55,25,54,55$ \\
$\mathrm{~B}$ & I, II, III, IV & $55,55,26,53$ \\
$\mathrm{C}$ & I, II, III, IV, V, VI & $50,21,38,24,55,55$ \\
$\mathrm{~N}$ & I, II, III, IV & $36,50,32,39$ \\
$\mathrm{O}$ & I, II, III, IV & $50,29,25,36$ \\
$\mathrm{~F}$ & I, II, III, IV & $50,50,50,50$ \\
$\mathrm{Ne}$ & I, II, III, IV & $50,50,50,50$ \\
$\mathrm{Na}$ & I, II, III, IV & $32,50,50,50$ \\
$\mathrm{Mg}$ & I, II, III, IV & $50,32,50,50$ \\
$\mathrm{Al}$ & I, II, III, IV & $41,50,31,50$ \\
$\mathrm{Si}$ & I, II, III, IV & $50,51,50,31$ \\
$\mathrm{~S}$ & I, II, III, IV & $50,50,50,50$ \\
$\mathrm{Ar}$ & I, II, III, IV & $50,50,50,50$ \\
$\mathrm{Ca}$ & I, II, III, IV & $50,17,50,50$ \\
\hline $\mathrm{Iron}$ & project & \\
$k$ & $j$ & $n_{i j}$ \\
\hline $\mathrm{Fe}$ & I, II, III & $50,50,50$ \\
\hline
\end{tabular}

not always occur at the observed frequencies. In STERNE we have tabulated the observed photoionization edges separately and imposed a cutoff on the cross-section for photon energies below the threshold.

\subsection{Bound-free absorption: IRON project cross-sections}

Photoionization cross-sections for the low ionization stages of iron were computed by the Opacity Project but have since been found to be of insufficient accuracy (Bautista 1997). New calculations for Fe I, Fe II, Fe III, Fe IV and Fe V are being carried out by the Ohio State University group, in collaboration with the IRON Project. The new ground state Fe I cross-sections are up to three orders of magnitude higher (Bautista \& Pradhan 1995), Fe II cross-sections are up to an order of magnitude higher (Nahar \& Pradhan 1994), and Fe III cross-sections are up to a factor of five lower (Nahar 1996) than the OP data. These new cross-sections for Fe I, II, and III have been incorporated into STERNE using the same methods we employed for the OP cross-sections.

\subsection{Bound-bound absorption: opacity sampling}

A major problem presented by the ODF approach is that hydrogen-deficient stars exhibit surfaces with highly-evolved chemical compositions and they can only be poorly characterised by a single mixture. Rather, they exhibit a range in average metallicity from 2 dex below solar to super-solar abundances, and large enhancements in carbon, nitrogen and oxygen. There are no two extreme helium stars with identical chemical compositions. Moreover, evolved stars show residual hydrogen abundances from normal down to less than 1 part per $10^{4}$.
An opacity sampling procedure has been implemented which accounts for contributions of up to $10^{6}$ atomic lines. Whereas ODFs are only available for a few selected hydrogendeficient mixtures, opacity sampling (OS) allows individual stellar abundances to be taken into consideration properly. As well, OS proves to be more flexible and adaptable than ODFs, as new values of oscillator strengths or new line lists can be easily incorporated without the need to recalculate extensive sets of opacity tables.

The opacity of a single line depends on the population of the absorber, the intrinsic strength of the transition - the oscillator strength or $f$-value, and the line profile. At frequency $v$ and optical depth $\tau$, the opacity due to line $i$ is represented by

$\kappa_{\mathrm{bb}, i}(v, \tau)=\mathcal{N}_{i}(\tau) \cdot \alpha_{0_{i}}\left(v_{i}, \tau\right) \cdot \Phi\left(v-v_{i}, \tau\right)$

where

$\alpha_{0}\left(v_{i}, \tau\right)=\frac{\pi e^{2}}{m_{\mathrm{e}} c} \frac{f}{\sqrt{\pi} \Delta v_{\mathrm{D}}}$.

Defining $v_{0}$ as the frequency measured from line centre $\left(v_{i}\right)$ in units of Doppler half-width:

$v_{0}(\tau)=\left|v-v_{i}\right| / \Delta v_{\mathrm{D}}(\tau)$

$\Delta \nu_{\mathrm{D}}(\tau)=\frac{v_{i}}{c}\left(\frac{2 k T(\tau)}{m_{k}}+\xi^{2}\right)^{1 / 2}$.

The atomic mass of species $k$ is $m_{k}$ and $\xi$ the microturbulent velocity. For the line profile, we use the Voigt function,

$\Phi\left(v-v_{i}, \tau\right) \equiv H\left(a(\tau), v_{0}(\tau)\right)$

where $a(\tau)$ represents the scaled line-broadening coefficients. Omitting $\tau$,

$a=\frac{\gamma_{\mathrm{r}}+n_{\mathrm{e}} \gamma_{\mathrm{s}}+n_{\mathrm{H}} \gamma_{\mathrm{w}}}{4 \pi \Delta v_{\mathrm{D}}}$

in which $\gamma_{\mathrm{r}}, \gamma_{\mathrm{s}}, \gamma_{\mathrm{w}}$ are the radiative, Stark and Van der Waal's damping constants and $n_{\mathrm{e}}, n_{\mathrm{H}}$ are the electron and hydrogen number densities, respectively.

The absorption coefficient per line and per absorber is then

$\alpha(v)=\alpha_{0} H\left(a, v_{0}\right)$.

Correcting the absorption for stimulated emission and multiplying by the number of absorbers in the lower state of the transition, we obtain the contribution from a given line

$$
\begin{aligned}
\alpha(v)= & \frac{\sqrt{\pi} e^{2}}{m_{\mathrm{e}} c \Delta v_{\mathrm{D}}}[1-\exp (-h v / k T)] \\
& \times \exp \left(-\chi_{\mathrm{L}} / k T\right) g_{\mathrm{L}} f_{\mathrm{LU}} H\left(a, v_{0}\right)
\end{aligned}
$$

where $f_{\mathrm{LU}}$ is the oscillator strength for a transition from a lower state $\mathrm{L}$ to upper state $\mathrm{U}$, and $\chi_{\mathrm{L}}, g_{\mathrm{L}}$ are the excitation potential and statistical weight for the lower state. The total opacity at any selected frequency $v$ is then the sum of the linear absorption coefficients due to each individual line at that frequency:

$$
\begin{aligned}
\alpha_{\mathrm{bb}}(v)= & \frac{\pi^{1 / 2} e^{2}}{m c}[1-\exp (-h v / k T)] \\
& \times \sum_{j k} \frac{\beta_{j k}}{G_{j k} \Delta v_{\mathrm{D} k}} \\
& \times \sum_{\mathrm{L}} \exp \left(-\chi_{\mathrm{L}} / k T\right) g_{\mathrm{L}} f_{\mathrm{LU}} \\
& \times H\left(a, v_{0}\right)
\end{aligned}
$$


Here the sum over $\mathrm{L}$ is the sum over all lines of ion $j$ of atom $k$ in a specified small frequency interval centered on $v$, and the sum over $j k$ is the sum over all ions $j$ of all atoms $k . \beta_{j k}$ and $G_{j k}$ represent the fractional abundances and partition functions of ion $j k$ respectively. The subscript $k$ in $\Delta v_{\mathrm{D} k}$ recognises that the Doppler half-width is a function of the contributing atom $\left(m_{k}\right)$. No averaging is performed; the contribution from each individual line is added in the calculation of the opacity at frequency $v$.

This summation must be carried out at every depth point and frequency point in the model atmosphere calculation, and many lines may contribute at each depth point. Consequently the implementation of the method in a computer code must be extremely efficient. We have devised a procedure, loosely based on Sneden et al. (1976), which aims to minimize the number of cpu clock cycles by decomposing the various terms into depthdependent and frequency-dependent quantities, as follows:

$v_{\mathrm{L}}^{\prime}=\log \left(\left|v-v_{\mathrm{L}}\right|\right)$

$\chi_{\mathrm{L}}^{\prime}=\chi_{\mathrm{L}}$

$\phi_{\mathrm{L}}^{\prime}=\ln \left(g_{\mathrm{L}} f_{\mathrm{LU}}\right)$

which only need to be established when reading in the linelist, providing that the reference frequencies $v$ used in the model calculation are specified. In addition, for ion $j$ of atom $k$,

$\Delta v_{\mathrm{D} k}=\frac{v}{c}\left(\frac{2 k T}{m_{k}}+\xi^{2}\right)^{1 / 2}$

$\Delta_{k}^{\prime}=\log \left(\Delta v_{\mathrm{D} k}\right)$

$\theta=1 / k T$

$\beta_{j k}^{\prime}=\ln \left(\frac{\beta_{j k}}{G_{j k} / \Delta \nu_{\mathrm{D} k}}\right)$

$S^{\prime}=\frac{\pi^{1 / 2} e^{2}}{m c}[1-\exp (-h v / k T)]$

are specified once for each frequency and depth point, but are independent of the line properties. To achieve this, we have introduced the approximation $v \approx v_{i}$ into (16) to improve efficiency. This is justified since lines are only considered to contribute to the local opacity if $|v|$ is smaller than some limit, e.g. $10^{-4}$.

In the Doppler approximation, where radiative and collisional damping are neglected, we may write

$H\left(a, v_{0}\right) \approx \exp \left(-v_{0}^{2}\right)$

and the total line opacity can be computed very cheaply since Eq. (12) may then be written as:

$\alpha_{\mathrm{bb}}(v)=S^{\prime} \sum_{\mathrm{L} j k} \exp \left(\beta_{j k}^{\prime}+\phi_{\mathrm{L}}^{\prime}-\chi_{\mathrm{L}}^{\prime} \theta-\nu_{\mathrm{L}}^{\prime \prime} \Delta_{k}^{\prime \prime}\right)$

where

$v_{\mathrm{L}}^{\prime \prime}=\left(v-v_{\mathrm{L}}\right)^{2}$

$\Delta_{k}^{\prime \prime}=1 /\left(\Delta v_{\mathrm{D} k}\right)^{2}$.

The contribution of each line to the sum then requires only two multiplications, three additions and one exponentiation.

However simple tests with $a=0.001$ show that the nonthermal contribution to the line profile is very important, especially in the ultraviolet. This can be dealt with, in a statistical approach, by tabulating the Voigt function or, rather, its logarithm, for carefully chosen values of $a, v_{0}$ :

$H_{p q}^{\prime}=\ln H\left(a_{p}, v_{0 q}\right) ; \quad p=0, n_{p}, \quad q=0, n_{q}$.

Equation (12) may then be written

$\alpha_{\mathrm{bb}}(v)=S^{\prime} \sum_{\mathrm{L} j k} \exp \left(\beta_{j k}^{\prime}+\phi_{\mathrm{L}}^{\prime}-\chi_{\mathrm{L}}^{\prime} \theta+H_{p q}^{\prime}\right)$

where integers $p, q$ are related to $a, v_{0}$ by:

$p=\left(\log (a)-p_{0}\right) n_{p} / \delta_{p}$

$q=\left(\log \left(v_{0}\right)-q_{0}\right) n_{q} / \delta_{q}$

where $n_{p, q}, \delta_{p, q}, p_{0}, q_{0}$ are the number range and zero-point values for which $H_{p q}^{\prime}$ is tabulated. In practice, these indices will be evaluated from physical quantities by

$$
\begin{aligned}
& p=\operatorname{nint}\left(\frac{n_{p}}{\delta_{p}}\left(\Gamma_{\mathrm{L}}^{\prime}+N_{d}^{\prime}-\Delta_{k}^{\prime}-p_{0}\right)\right) \\
& q=\operatorname{nint}\left(\frac{n_{q}}{\delta_{q}}\left(v_{\mathrm{L}}^{\prime}-\Delta_{k}^{\prime}-q_{0}\right)\right)
\end{aligned}
$$

where the damping constant $\Gamma_{\mathrm{L}}^{\prime}$ is chosen from one of three approximations

$\begin{array}{ll}\Gamma_{\mathrm{L}}^{\prime}=\log \left(\gamma_{\mathrm{r}} / 4 \pi\right) & N_{d}^{\prime}=0 \\ \Gamma_{\mathrm{L}}^{\prime}=\log \left(\gamma_{\mathrm{s}} / 4 \pi\right) & N_{d}^{\prime}=\log \left(n_{\mathrm{e} d}\right) \\ \Gamma_{\mathrm{L}}^{\prime}=\log \left(\gamma_{\mathrm{w}} / 4 \pi\right) & N_{d}^{\prime}=\log \left(n_{\mathrm{H} d}\right)\end{array}$

where $n_{\ldots d}$ represents the appropriate particle density at depth point $d$ and the $\gamma$ 's refer to a particular line $L$. The choice of approximation must be made with care in order to avoid unnecessary computation but without loss of accuracy. One example is the case of hot stars where Van der Waal's broadening (31) is negligible compared with electron broadening (30). For each line the maximum contribution for damping is selected.

Having established $\alpha$, the absorption coefficient per atom, we obtain the line-absorption coefficient per gram $\kappa$ from

$\kappa_{\mathrm{bb}}(v)=\frac{N_{\mathrm{A}}}{\mu_{\mathrm{I}}} \alpha_{\mathrm{bb}}(v)$.

Computed in this fashion, the opacity sampling method is able to account for the depth-dependence of the variation with frequency of the line opacities. The method can also account for a change in composition simply by changing the model composition parameters.

\subsection{Wavelength grid and line selection}

The wavelength grid may be adjusted to allow more or less structure in the opacity to be included. To treat the photoionization cross-sections including the PEC resonances in detail, the grid should consist of a very high number of wavelength points. This would lead to unacceptable demands for computing time. In contrast, relatively few wavelength points are required to obtain smooth cross-sections which exclude the detailed structure of the PEC resonances. Since such smoothing preserves the overall cross-sections to within an error of $10 \%$, 


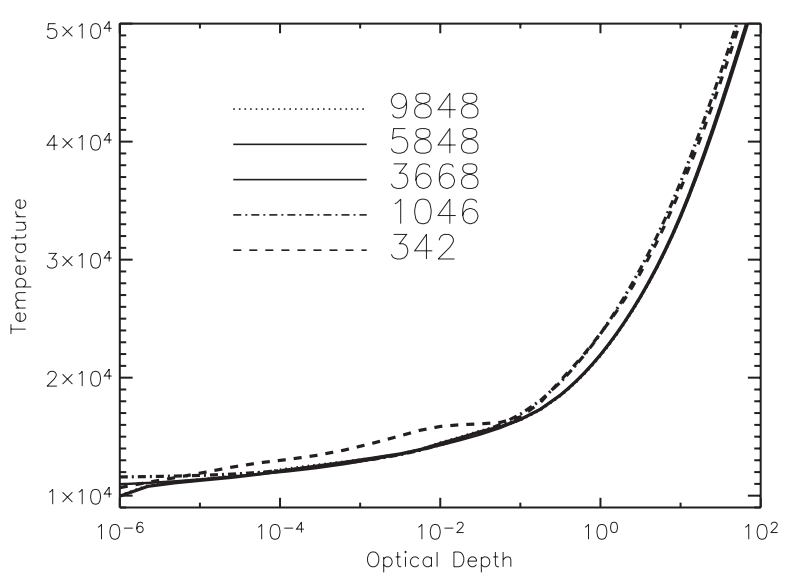

Fig. 1. Temperature distributions for a hydrogen-rich atmosphere with $T_{\text {eff }}=20000 \mathrm{~K}$ calculated using models with different numbers of wavelength grid points.

we have chosen not to follow the PEC resonance structure in detail.

Similarly, including the opacity contribution from all absorption lines demands a large number of wavelength points. The wavelength grid must guarantee the selection of dominant lines in the photosphere and must also account for the contribution of weak lines in a statistically correct manner. If considering both atomic and molecular lines then 20 million lines or more need to be included, and additional line selection at each grid point must be carried out to keep the number of lines within reason. However for hot stars, only atomic lines need to be accounted for. There are approximately 600000 atomic lines in the Kurucz list (1995). Therefore line selection is governed solely by the definition of the wavelength grid.

The grid used for the models presented in this paper allows a finer spacing at shorter wavelengths, increasing in coarseness with increasing wavelength. The chosen grid uses 3668 wavelength points, spaced by $0.5 \AA$ at $\lambda<500 \AA, 1.0 \AA$ for $500<$ $\lambda<900 \AA, 2.5 \AA$ for $900<\lambda<5000 \AA$, and at larger intervals thereafter. The spacing of the points is chosen in such a way that the emphasis is placed on the areas in the spectrum where most of the flux emerges and where most of the lines are found. To insure that important lines are not missed, additional points were added at the positions of known strong lines, for example the Balmer lines. The amount of time needed for each iteration increases with the number of wavelength points added, as the transfer equation must be solved at each wavelength point. The optimal number of wavelength points $(n)$ was determined by demanding that the relative temperature difference between one model and that computed with approximately double the amount of grid points should be less than $1 \%$. Temperature distributions for a hydrogen-rich atmosphere with $T_{\text {eff }}=20000 \mathrm{~K}$ computed using different grids is shown in Fig. 1. Relative temperature differences $\left(T_{3668}-T_{n}\right) / T_{3668}$ with respect to the chosen model are shown in Fig. 2, where $T_{n}$ is the temperature computed with $n$ wavelength points.

In order to minimize the computational cost of producing OS models, the wavelength grid should become a function of effective temperature. Absorption lines to be included would

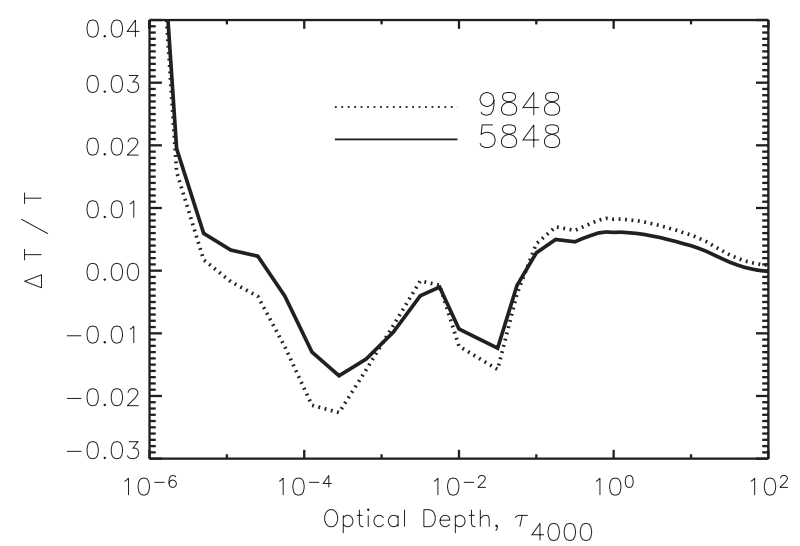

Fig. 2. Relative temperature differences $\left(T_{3668}-T_{n}\right) / T_{3668}$ with respect to the chosen reference model with 3668 wavelength points.

then be preselected using a starting approximation. Future versions of STERNE will include an automatic grid selection based on the input parameters, and will be more uniformly spaced in frequency, rather than wavelength.

\section{Model calculations}

By introducing these revisions to the opacities, we have defined a major new version of STERNE. To distinguish this version, we have labelled the old version based on Kurucz and Peach opacity tables and ODF line opacity, STERNE2. The new version based on Opacity Project photoionization and opacity sampling, is labelled STERNE3. In order to test the effect of the new opacities we have computed a number of models across a range of effective temperature $T_{\text {eff }}=10,20,30$ and $40 \mathrm{kK}$, and for fractional hydrogen abundances $n_{\mathrm{H}}=0.911$ and 0.00 with both versions of STERNE. Since it is possible to switch off the line opacity completely, we have computed models with and without line opacity in order to compare the effect of changing the continuous opacities alone. We have also calculated a set of models to compare directly with the grid of ATLAS9 model atmospheres published by Kurucz (1991).

\section{Model comparisons}

In general, evaluations have been made by comparing the emergent flux distributions over the entire UV - visible range. Since the flux varies by several orders of magnitude in this range, we have also compared the ratios of fluxes. A third test is provided by the comparing the temperature $T_{\tau}$ as a function of optical depth. Finally, it would be prohibitive to show the difference in opacity contribution for all ion species over all temperatures and compositions; however we do demonstrate the major differences between the new and old photoionization cross-sections.

\subsection{STERNE3 vs. ATLAS9}

A comparison of the STERNE3 models with ATLAS9 models (Kurucz 1991) has been carried out using a solar composition for four $T_{\mathrm{eff}}, \log g$ pairs: $(10000 \mathrm{~K}, 3.00),(20000 \mathrm{~K}, 3.00)$, $(30000 \mathrm{~K}, 3.50)$ and $(40000 \mathrm{~K}, 4.50)$. The models have been 

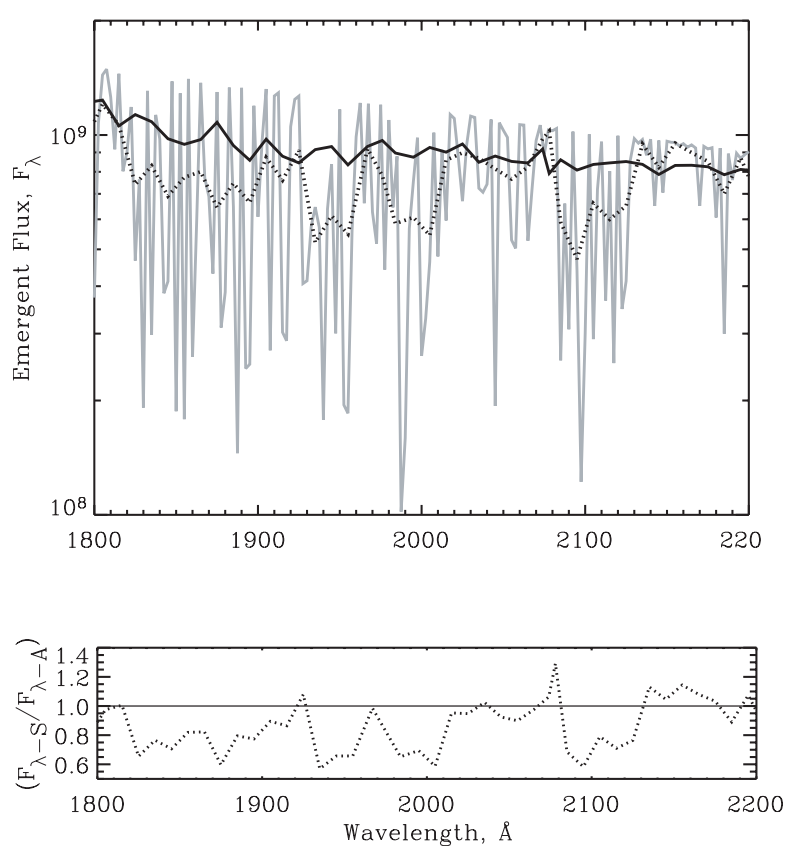

Fig. 3. The local effect of the dense line spectrum due to the iron group elements on the flux distribution of a solar composition model with $T_{\text {eff }}=20000 \mathrm{~K}$ and $\log g=3.00$ is shown. The ATLAS9 model is shown by the solid line. The STERNE3 model is shown by the grey line, and is resampled to the ATLAS9 model grid for comparison (dotted line).

found to agree very well. Differences in the models are due to (i) the use of different standard values for the solar abundances (Kurucz uses values from Anders \& Grevesse (1989), while STERNE3 has adopted the values as given by Grevesse \& Sauval (1998)), ii) the opacity sampling approach which retains more structure, even in the resampled version, and iii) differences in the continuous opacities. The local effect of the dense line spectrum due to iron group elements at approximately $2000 \AA$, appears to be much stronger when using the OS method than when using the ODF method used in ATLAS9, although it seems to have little effect on the gradient of the optical continuum (Fig. 3).

\subsection{STERNE3 vs. STERNE2: continuous opacity}

Direct comparisons of the old and new photoionization crosssections were performed. Studying the effects of the new opacities on the model structure, minor differences were found for hydrogen-rich atmospheres, while significant differences were found for helium-rich atmospheres. A full description, including text and figures, is available in the online version of this paper.

\subsection{STERNE3 vs. STERNE2: line opacity}

A comparison of continuum plus line opacity STERNE3 and STERNE2 models for a fixed hydrogen-rich composition and a helium-rich composition was carried out. Modest temperature increases were found in the line forming region of the hydrogen-rich atmospheres. The temperature increases by approximately $200 \mathrm{~K}$ for $T_{\text {eff }}=10000 \mathrm{~K}$ and $20000 \mathrm{~K}$. Increases of up to $1250 \mathrm{~K}$ were seen for $T_{\text {eff }}=30000 \mathrm{~K}$, and $2000 \mathrm{~K}$ for $T_{\text {eff }}=40000 \mathrm{~K}$. Higher temperature increases were found in the line forming region of the helium-rich atmosphere. Increases in temperature of $1000 \mathrm{~K}$ were found for $T_{\text {eff }}=10000 \mathrm{~K}$ and $20000 \mathrm{~K}, 3000 \mathrm{~K}$ for $T_{\text {eff }}=30000 \mathrm{~K}$ and up to $3500 \mathrm{~K}$ for $T_{\text {eff }}=40000 \mathrm{~K}$. A full description, including text and figures, is available in the online version of this paper.

\section{Summary and conclusions}

We have completely revised the treatment of opacity in the stellar atmosphere code STERNE. Opacity Project and IRON Project photoionization cross-sections have been used to compute the continuous opacity, and line opacity has been treated using an opacity sampling procedure.

A comparison of this new version (STERNE3) with STERNE2, which used old continuous opacity tables and opacity distribution functions, has been carried out. We have highlighted the impact of the new opacities on the atmospheric structure, particularly in the hydrogen-deficient case. Significant increases in the carbon and nitrogen opacities were found when using the OP cross-sections. These increases in opacity are reflected in the flux distribution of $\mathrm{H}$-deficient stars and will be important in the analysis of stars with enriched carbon and nitrogen, as in the case of extreme helium stars. Paper II will apply the new models to these stars.

The effects of the new continuous opacities and opacity sampling are not restricted to the hydrogen-deficient case. We have found that the temperature distributions of the new models have on average hotter line forming regions compared to the old models. Increases of up to $1250 \mathrm{~K}$ are seen in the hydrogenrich models while increase of up to $3000 \mathrm{~K}$ are seen in heliumrich models. These temperature increases will have implications on effective temperature measurements, particularly for hot stars, and may help to explain discrepancies between flux fitting methods and ionization measures of $T_{\text {eff }}$.

Allowing elemental abundances to vary in the STERNE3 models will prove extremely useful in situations where the object's atmospheric composition differs significantly from the solar composition, such as in the case of chemically peculiar stars, metal-poor stars with significant $\alpha$-element enhancements and extreme helium stars. The opacity sampling approach will also allow the examination of the effects of elemental stratification on the atmospheric structure, applicable to stars which display diffusion effects.

Acknowledgements. Research at Armagh Observatory is funded by the Department of Culture, Arts and Leisure, Northern Ireland. We are indebted to Mr P.M. Harrison, formerly at the University of St Andrews, for developing a part of the new photoionization software, and to Ulrich Heber for his helpful comments and suggestions in improving this paper. 
Table A.1. Coefficients for Rayleigh scattering for H: Dalgarno (1962) and for He, C, N and O: Tarafdar \& Vardya (1969).

\begin{tabular}{lcccr}
\hline \hline$k$ & $a_{k}$ & $b_{k}$ & $c_{k}$ & $\lambda_{\mathrm{R} k} / \AA$ \\
\hline $\mathrm{H}$ & $5.799 \times 10^{-13}$ & $2.452 \times 10^{6}$ & $4.801 \times 10^{12}$ & 1215 \\
$\mathrm{He}$ & $5.292 \times 10^{-14}$ & $0.470 \times 10^{6}$ & $0.182 \times 10^{12}$ & 584 \\
$\mathrm{C}$ & $4.288 \times 10^{-12}$ & $2.671 \times 10^{6}$ & $6.562 \times 10^{12}$ & 1657 \\
$\mathrm{~N}$ & $2.319 \times 10^{-12}$ & $1.995 \times 10^{6}$ & $3.362 \times 10^{12}$ & 1200 \\
$\mathrm{O}$ & $7.585 \times 10^{-13}$ & $0.997 \times 10^{6}$ & $1.101 \times 10^{12}$ & 1302 \\
\hline
\end{tabular}

\section{Appendix A: Scattering}

\section{A.1. Thomson \& Rayleigh scattering}

The scattering of light by free electrons is referred to as Thomson scattering, for which the classical formula

$\sigma_{\mathrm{e}}=\left(8 \pi e^{4} / 3 m^{2} c^{4}\right)=6.65 \times 10^{-25} \mathrm{~cm}^{2}$

gives the cross-section per electron and is independent of frequency.

The scattering of radiation by bound systems, such as atoms and molecules, at frequencies much lower than the characteristic transition frequencies of the system, is known as Rayleigh scattering, $\sigma_{\mathrm{R}}$. For frequencies $v \ll v_{i j}$ and characteristic resonant frequencies with strengths $f_{i j}$,

$\sigma_{\mathrm{R} i j}(v)=\sigma_{\mathrm{e}} f_{i j} v^{4} /\left(v_{i j}^{2}-v^{2}\right)^{2}$.

Following Tarafdar \& Vardya (1969), this is computed from

$\sigma_{\mathrm{R} k}(\lambda)=a_{k} / \lambda^{4}\left(1+b_{k} / \lambda^{2}+c_{k} / \lambda^{4}\right) g_{0 k} / u_{k}, \lambda>\lambda_{\mathrm{R} k}$

where $a_{k}, b_{k}, c_{k}$ are given for each scatterer $k$ (Table A.1) with partition functions $u_{k}$ and ground-state statistical weights $g_{0 k}$ for wavelengths greater than the characteristic wavelength, $\lambda_{\mathrm{R} k}$. Rayleigh scattering coefficients due to $\mathrm{H}, \mathrm{He}, \mathrm{C}, \mathrm{N}$, and $\mathrm{O}$ are included in STERNE.

The total opacity per unit mass due to scattering is then given by

$\sigma_{v}=\frac{N_{\mathrm{A}}}{\mu_{\mathrm{I}}}\left(\sigma_{\mathrm{e}}+\sum_{k} \beta_{k} \sigma_{\mathrm{R} k}(v)\right)$

where $\beta_{k}$ corresponds to the fractional abundance of neutral ions of species $k\left(\sum_{j k} \beta_{j k}=1\right) . N_{\mathrm{A}}$ and $\mu_{\mathrm{I}}$ refer to Avogadro's number and the mean atomic mass per atom respectively.

\section{A.2. Free-free absorption}

For radiation at frequencies much closer to the characteristic frequencies, photons are scattered by free electrons passing near a bound system because of the creation of a transitory dipole moment. Cross-sections for this process in hydrogen are given by

$\alpha_{\mathrm{ff}}(v, T)=\left(\frac{4 e^{6}}{3 c h}\right)\left(\frac{2 \pi}{3 k m^{3}}\right)^{\frac{1}{2}} \frac{\overline{g_{\mathrm{ff}}}(v, T)}{v^{3} \sqrt{T}}$

where $\overline{g_{\mathrm{ff}}}$ is the thermal average of the Gaunt factor (Gingerich 1964; Karzas \& Latter 1961)

$\overline{g_{\mathrm{ff}}}(v, T) \equiv \int_{0}^{\infty} g_{\mathrm{ff}}(v, v) \mathrm{e}^{-u} \mathrm{~d} u$ and $u \equiv\left(m v^{2} / 2 k T\right)$. Evaluating the constants we obtain,

$\alpha_{\mathrm{ff}}(v, T)=\frac{3.694 \times 10^{8} \overline{g_{\mathrm{ff}}}(v, T)}{v^{3} \sqrt{T}}$.

If the free electrons are known to have a Maxwellian distribution, these calculations may be extended to include hydrogenic free-free absorption. Photoionization of multi-electron ions is decidedly non-hydrogenic. However, since very few experimental results are available, it is necessary to choose a theoretical approximation. Corrected for stimulated emission, the hydrogenic absorption cross-section per ion and per electron is,

$\alpha_{\mathrm{ff}}(v, T)=\frac{3.694 \times 10^{8} \overline{g_{\mathrm{ff}}}(v, T) Z^{2}}{v^{3} \sqrt{T}}\left(1-\mathrm{e}^{-\frac{h \nu}{k T}}\right)$

where $Z$ is the atomic number of the species.

\section{References}

Anders, E., Grevesse, N. 1989, Geochim. Cosmochim. Acta, 53, 197 Anderson, L., \& Grigsby, J. A. 1991, in Stellar Atmospheres: Beyond Classical Models, ed. L. Crivellari, I. Hubeny, \& D. G. Hummer, NATO ASI Ser. (Dordrecht: Kluwer), 365

Asplund, M., Gustafsson, B., Kiselman, D., \& Eriksson, K. 1997, A\&A, 318, 521

Bautista, M. A. 1997, A\&AS, 122, 167

Bautista, M. A., \& Pradhan, A. K. 1995, J. Phys. B, 28, L176

Berrington, K. 1995, in Astrophysical Applications of Powerful New Databases, ed. S. J. Adelman, \& W. L. Wiese, ASP Conf. Ser., 78, 19

Böhm, K. H., \& Deinzer, W. 1965, Z. Astrophys., 61, 1

Burgess, A., \& Seaton, M. J. 1960, MNRAS, 120, 121

Burke, V. M., \& Lennon, D. J. 1996, Data available at TOPbase

Cunto, W., Mendoza, C., Ochsenbein, F., \& Zeippen, C. J. 1993, A\&A, 275, L5

Dalgarno, A. 1962, Geophys. Corp. of America, GCA Tech. Rep., \#62-28-A

Fernley, J. A., Taylor, K. T., \& Seaton, M. J. 1987, J. Phys. B, 20, 6457

Fernley, J. A., Hibbert, A., Kingston, A. E., \& Seaton, M. J. 1999, J. Phys. B, 32, 5507

Gingerich, O. 1964, SAOSR, 167, 17

Grevesse, N., \& Sauval, A. J. 1998, Space Sci. Rev., 85, 161

Gustafsson, B., Bell, R. A., Eriksson, K., \& Nordlund, A. 1975, A\&A, 42,407

Hauschildt, P. H. 1992, JQSRT, 47, 433

Heber, U. 1984, A\&A, 130, 119

Heber, U., \& Schönberner, D. 1981, A\&A, 102, 73

Hubeny, I., \& Lanz, T. 1995, ApJ, 439, 875

Hummer, D. G., Berrington, K. A., Eissner, W., et al. 1993, A\&A, 279, 298

Hunger, K., \& Van Blerkom, D. 1967, Z. Astrophys., 66, 185

Jeffery, C. S. 1989, QJRAS, 30, 195

Jeffery, C. S., \& Heber, U. 1992, A\&A, 260, 133

Jeffery, C. S., \& Heber, U. 1994, Sterne: User Guide, CCP7 Software Library, http://ccp7.dur.ac.uk/library.html

Jeffery, C. S., Woolf, V. M., \& Pollacco, D. L. 2001, A\&A, 376, 497

Karzas, W. J., \& Latter, R. 1961, ApJS, 6, 167

Koester, D., Vauclair, G., Dolez, N., et al. 1985, A\&A, 149, 423

Kurucz, R. L. 1970, SAOSR, No. 309

Kurucz, R. L. 1991, in Stellar Atmospheres: Beyond Classical Models, NATO ASI Ser., 341, 441

Kurucz, R. L. 1993, Kurucz CD-ROM 13: ATLAS9, SAO, Cambridge, USA 
Kurucz, R. L. 1996, in Model Atmospheres and Spectrum Synthesis, ed. S. J. Adelman, F. Kupka, \& W. W. Weiss (San Francisco: ASP), 160

Kurucz, R. L., \& Bell, B. 1995, Kurucz CD-ROM 23: Atomic Line List, SAO, Cambridge, USA

Kurucz, R. L., \& Peytremann, E. 1975, SAOSR, 326, 1

Luo, D., \& Pradhan, A. K. 1989, J. Phys. B, 22, 3377

Möller, R. U. 1990, Diplom. Thesis, Universität Kiel

Nahar, S. N. 1996, Phys. Rev. A, 53, 1545

Nahar, S. N., \& Pradhan, A. K. 1994, J. Phys. B, 27, 429

Pandey, G. P., Lambert, D. L., Jeffery, C. S., \& Rao, N. K. 2005, ApJ, submitted

Peach, G. 1967, MNRAS, 71, 13

Peach, G. 1970, MNRAS, 73, 1

Peytremann, E. 1974, A\&A, 33, 203

Przybilla, N., Butler, K., Heber, U., \& Jeffery, C. S. 2005, A\&A, submitted

Schönberner, D., \& Wolf, R. 1974, A\&A, 37, 87
Seaton, M. J., Yan, Y., Mihalas, D., \& Pradhan, A. K. 1994, MNRAS, 266, 805

Shulyak, D., Tsymbal, V., Ryabchikova, T., Stütz, Ch., \& Weiss, W. W. 2004, A\&A, 428, 993

Sneden, C., Johnson, H. R., \& Krupp, B. M. 1976, ApJ, 204, 281

Stewart, A. L. 1978a, J. Phys. B, 11, 2449

Stewart, A. L. 1978b, J. Phys. B, 11, L431

Stewart, A. L. 1979, J. Phys. B, 12, 401

Tarafdar, S. P., \& Vardya, M. S. 1969, MNRAS, 145, 171

The Opacity Project Team 1995, The Opacity Project, Vol. 1, Institute of Physics, Bristol

The Opacity Project Team 1997, The Opacity Project, Vol. 2, Institute of Physics, Bristol

Tully, J. A., Seaton, M. J., \& Berrington, K. A. 1990, J. Phys. B, 23, 3811

Werner, K. 1986, A\&A, 161, 177

Yan, Y., \& Seaton, M. J. 1987, J. Phys. B, 20, 6409 
N. T. Behara and C. S. Jeffery: LTE model atmospheres with new opacities, Online Material $p 1$

\section{Online Material}




\section{Appendix B:}

\section{B.1. STERNE3 vs. STERNE2: continuous opacity}

A comparison of the old and new photoionization crosssections for the ions He I, C I, C II, C III, N I, N II and N III is shown in Figs. B.1 and B.2. We computed the contribution to the total opacity in a hydrogen-deficient atmosphere with $T_{\text {eff }}=20000 \mathrm{~K}$ for each ion using both sets of cross-sections. The ratio of the new opacity to the old opacity is plotted as a function of wavelength at a fixed optical depth, $\tau=1$. The ratio over all depths as a function of wavelength is also plotted to show the behaviour throughout the atmosphere.

The contribution to the total opacity from the OP He I crosssections (Fernley et al. 1987) compared to the contribution from the old cross-sections is greater for all terms by up to $20 \%$ with the exception of the $1^{1} \mathrm{~S}$ term, where a decrease in opacity of up to $20 \%$ is found.

The opacity calculated using the OP cross-sections for C I (Luo \& Pradhan 1989), C II (Yan \& Seaton 1987) and C III (Tully et al. 1990) is greater at smaller wavelengths than the opacity computed using the old cross-sections. For C $\mathrm{I}$ the result is an increase in opacity of up to a factor of 10 for $\lambda<3300 \AA$. At longer wavelengths and deeper in the atmosphere, the old cross-sections are greater by up to $60 \%$. For C II a significant increase in opacity of up to a factor of 200 is found at $\lambda<1200 \AA$. At $\lambda>1200 \AA$, the old cross-sections produce approximately $40 \%$ more opacity. For C III significant increases of up to a factor of $10^{4}$ are found at all wavelengths where C III continuous opacity is computed.

The application of the OP cross-sections calculated for N I (Burke \& Lennon 1996), N II (Luo \& Pradhan 1989) and N III (Fernley et al. 1999) to the continuous opacity in STERNE results in significant increases. For N I an increase of up to a factor of 7 is found at $\lambda<3000 \AA$. The old cross-sections produce up to $50 \%$ more opacity for $\lambda>3000 \AA$. For N II a significant increase by up to a factor of 1000 is found at $\lambda<1200 \AA$. At longer wavelengths, the opacity drops to approximately $60 \%$ of the old opacity. For N III, increases in opacity by up to a factor of $10^{4}$ are found.

A comparison of the temperature structures of continuumonly STERNE3 and STERNE2 models for a fixed hydrogenrich composition and four effective temperatures is shown in Fig. B.2. The composition of the models is $n_{\mathrm{H}}=0.911, n_{\mathrm{He}}=$ 0.089 and solar metals with $\log g=3.00$. The models agree well at low temperatures, but begin to diverge at higher temperatures. These differences result from an increase in the continuous opacity at shorter wavelengths due primarily to ions which are not present in the STERNE2 model continuum sources.

A comparison of the temperature structures of continuumonly models with a helium-rich composition is shown inFig. B.2. The composition is $n_{\mathrm{H}}=0.00, n_{\mathrm{He}}=0.99$ and $n_{\mathrm{C}}=0.01$ with $\log g=3.00$. Discrepancies between the two models are found at all temperatures.
Increases in temperature deep in the atmosphere for $T_{\text {eff }}=$ $10000 \mathrm{~K}$ and $T_{\text {eff }}=20000 \mathrm{~K}$ are found in the new models. The primary source of the increase is the C II continuous opacity. An increase in C II opacity leads to an increase in the continuum opacity at $\lambda<1000 \AA$, resulting in more UV flux with a steeper continuum at $\lambda>1200 \AA$ (Fig. B.1). For $T_{\text {eff }}=30000 \mathrm{~K}$ and $T_{\text {eff }}=40000 \mathrm{~K}$ increases in temperature are found at smaller optical depth. These increases are due an increase in opacity at $\lambda<600 \AA$.

\section{B.2. STERNE3 vs. STERNE2: line opacity}

A comparison of continuum and line opacity STERNE3 and STERNE2 models for a fixed hydrogen-rich composition and varying effective temperature is shown in Fig. B.5. The STERNE3 model has been resampled on the STERNE2 wavelength grid for comparison. The composition of the models is $n_{\mathrm{H}}=0.911$ and $n_{\mathrm{He}}=0.089$, with $\log g=3.00$.

The low temperature models agree very well. For $T_{\text {eff }}=30000 \mathrm{~K}$ the STERNE3 model displays increased opacity in the regions $300 \AA<\lambda<400 \AA$ and $500 \AA<\lambda<600 \AA$. Increased opacity at $\lambda<400 \AA$ leads to a steeper continuum at $\lambda>600 \AA$ for $T_{\text {eff }}=40000 \mathrm{~K}$.

The effect of the new opacities on the temperature distribution in the atmosphere is shown in Fig. B.6. The difference in temperature $\left(T_{\mathrm{STERNE} 3}-T_{\mathrm{STERNE2}}\right)$ is shown as a function of optical depth. In the line forming region, approximated as roughly $10^{-2}<\tau<10^{0}$, the temperature increases by approximately $200 \mathrm{~K}$ for $T_{\text {eff }}=10000 \mathrm{~K}$ and $20000 \mathrm{~K}$. For $T_{\text {eff }}=30000 \mathrm{~K}$ the change in temperature ranges from $-500 \mathrm{~K}$ at smaller optical depth to $1250 \mathrm{~K}$ deeper in the atmosphere. For $T_{\text {eff }}=40000 \mathrm{~K}$ the range is $200 \mathrm{~K}$ to $2000 \mathrm{~K}$ for increasing optical depth.

A comparison of the two models with a helium-rich composition is shown in Fig. B.7. The composition of the models is $n_{\mathrm{H}}=0.00, n_{\mathrm{He}}=0.99$ and $n_{\mathrm{C}}=0.01$ with $\log g=3.00$. Discrepancies between the two models are found at all temperatures.

Absorption lines of the iron group elements are visible in the STERNE3 models at all temperatures around $2000 \AA$. Increased opacity at shorter wavelengths leads to a steeper continuum in all four models. For $T_{\text {eff }}=10000 \mathrm{~K}$ this occurs at $\lambda>4000 \AA$, for $20000 \mathrm{~K}$ at $\lambda>2000 \AA$, for $30000 \mathrm{~K}$ at $\lambda>1000 \AA$ and for $40000 \mathrm{~K}$ at $\lambda>600 \AA$. In all cases the total flux is conserved.

The effect of the new opacities on the temperature distribution is shown in Fig. B.8. The difference in temperature ( $\left.T_{\text {STERNE3 }}-T_{\text {STERNE2 }}\right)$ is shown as a function of optical depth. In the line forming region we see an increase in the temperatures of up to $3500 \mathrm{~K}$ for $T_{\text {eff }}=40000 \mathrm{~K}, 3000 \mathrm{~K}$ for $T_{\text {eff }}=30000 \mathrm{~K}$ and roughly $1000 \mathrm{~K}$ for $T_{\text {eff }}=20000 \mathrm{~K}$ and $10000 \mathrm{~K}$. 
N. T. Behara and C. S. Jeffery: LTE model atmospheres with new opacities, Online Material $p 3$
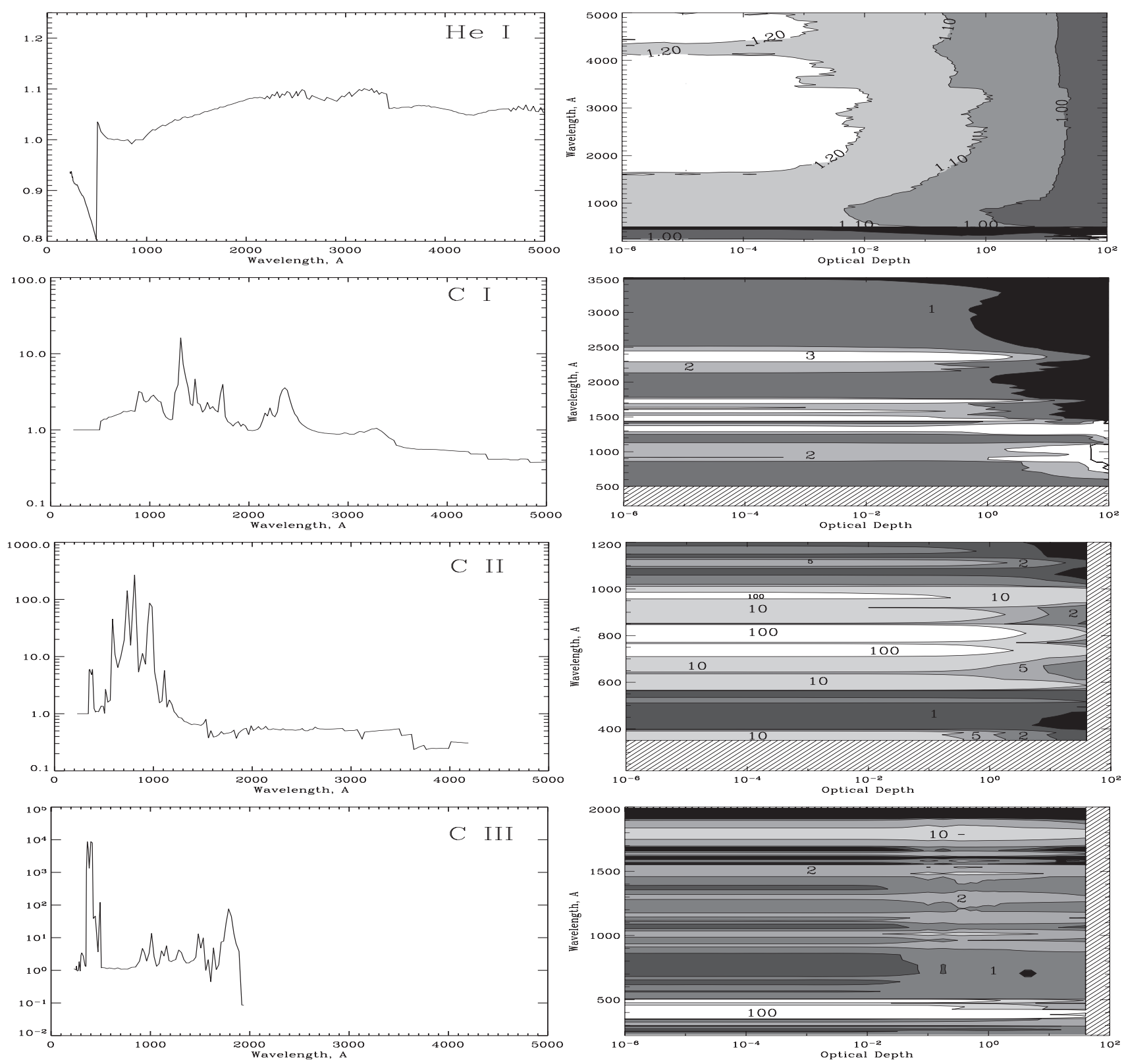

Fig. B.1. The ratio of the opacity computed using the STERNE3 cross-sections to the opacity computed using the STERNE2 cross-sections is plotted as a function of wavelength at optical depth $\tau=1$ for a hydrogen-deficient atmosphere with $T_{\text {eff }}=20000 \mathrm{~K}$. The contour plots on the right display the ratio as a function of wavelength and all optical depths in the atmosphere. Hatching denotes areas with no opacity data. 
N. T. Behara and C. S. Jeffery: LTE model atmospheres with new opacities, Online Material p 4
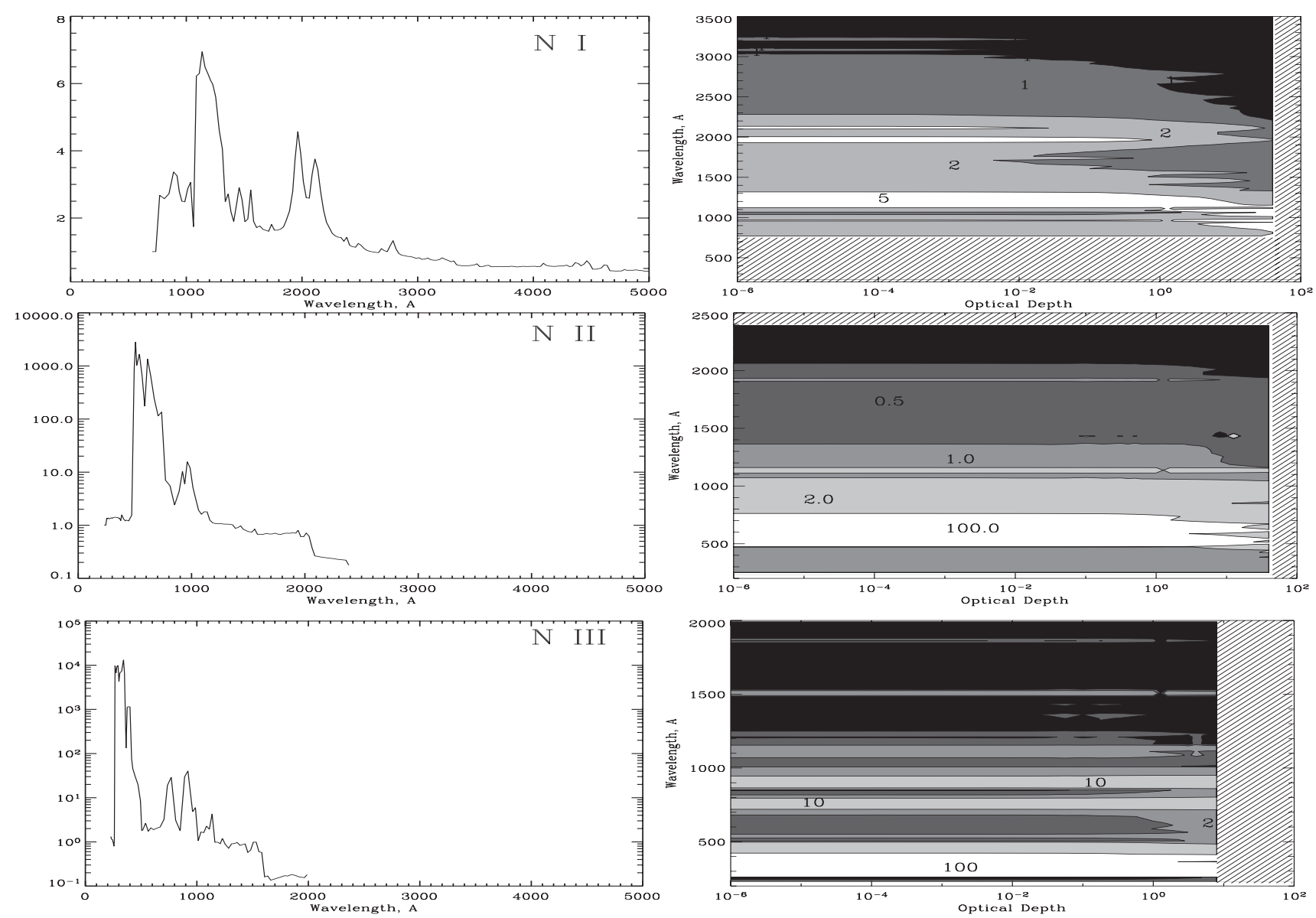

Fig. B.2. The ratio of the opacity computed using the STERNE3 cross-sections to the opacity computed using the STERNE2 cross-sections is plotted as a function of wavelength at optical depth $\tau=1$, for a hydrogen-deficient atmosphere with $T_{\text {eff }}=20000 \mathrm{~K}$ for N I and N II and $T_{\text {eff }}=30000 \mathrm{~K}$ for N III. The contour plots on the right display the ratio as a function of wavelength and all optical depths in the atmosphere. Hatching denotes areas with no opacity data.
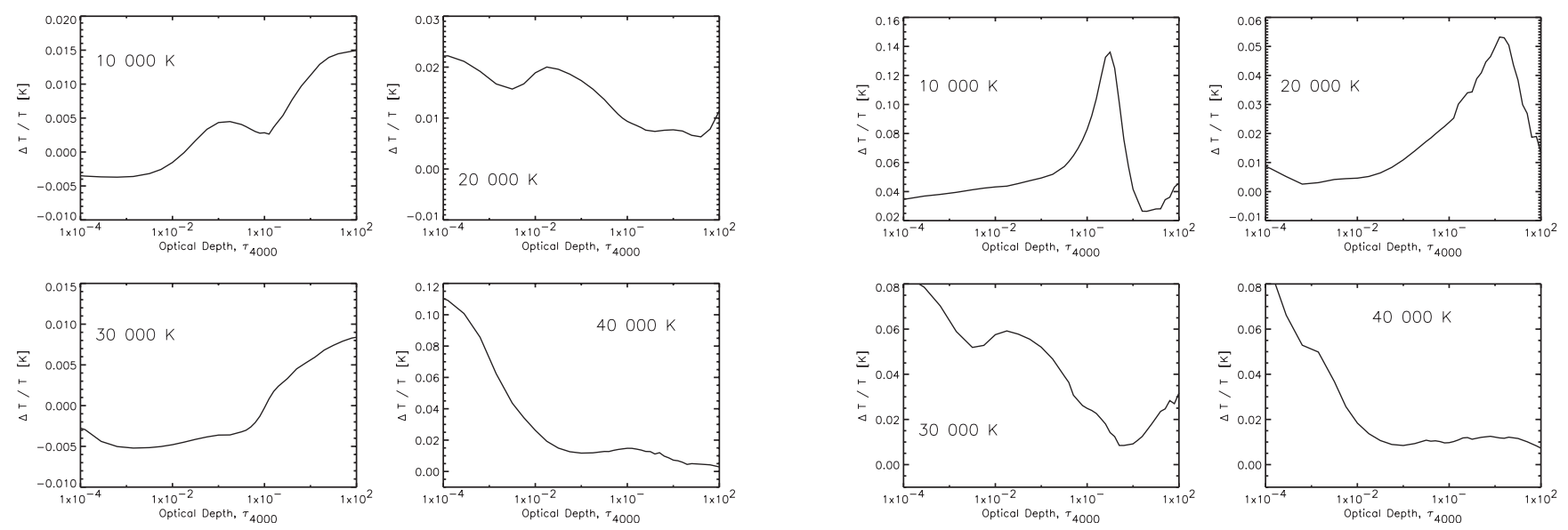

Fig. B.3.
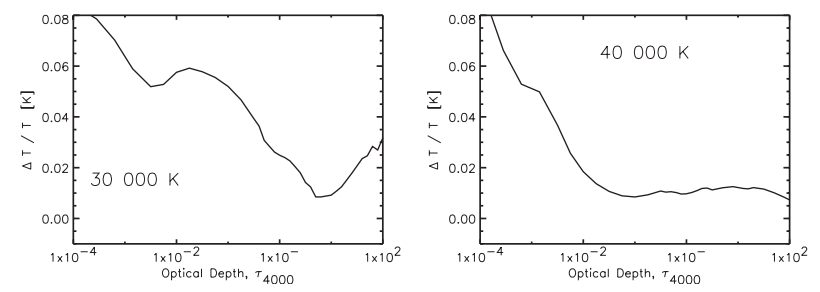

Fig. B.4.

Fig. B.3. (Left) relative temperature differences $\left(T_{S 3}-T_{S 2}\right) / T_{S 3}$ for continuum-only models with varying effective temperatures, $\log g=3.00$, $n_{\mathrm{H}}=0.911, n_{\mathrm{He}}=0.089$ and solar metals $(\mathrm{S} 3=$ STERNE3 and S2 $=$ STERNE2).

Fig. B.4. (Right) relative temperature differences $\left(T_{S 3}-T_{S 2}\right) / T_{S 3}$ for continuum-only models with varying effective temperatures, log $g=3.00$, $n_{\mathrm{H}}=0.00, n_{\mathrm{He}}=0.99$ and $n_{\mathrm{C}}=0.01$. 
N. T. Behara and C. S. Jeffery: LTE model atmospheres with new opacities, Online Material p 5
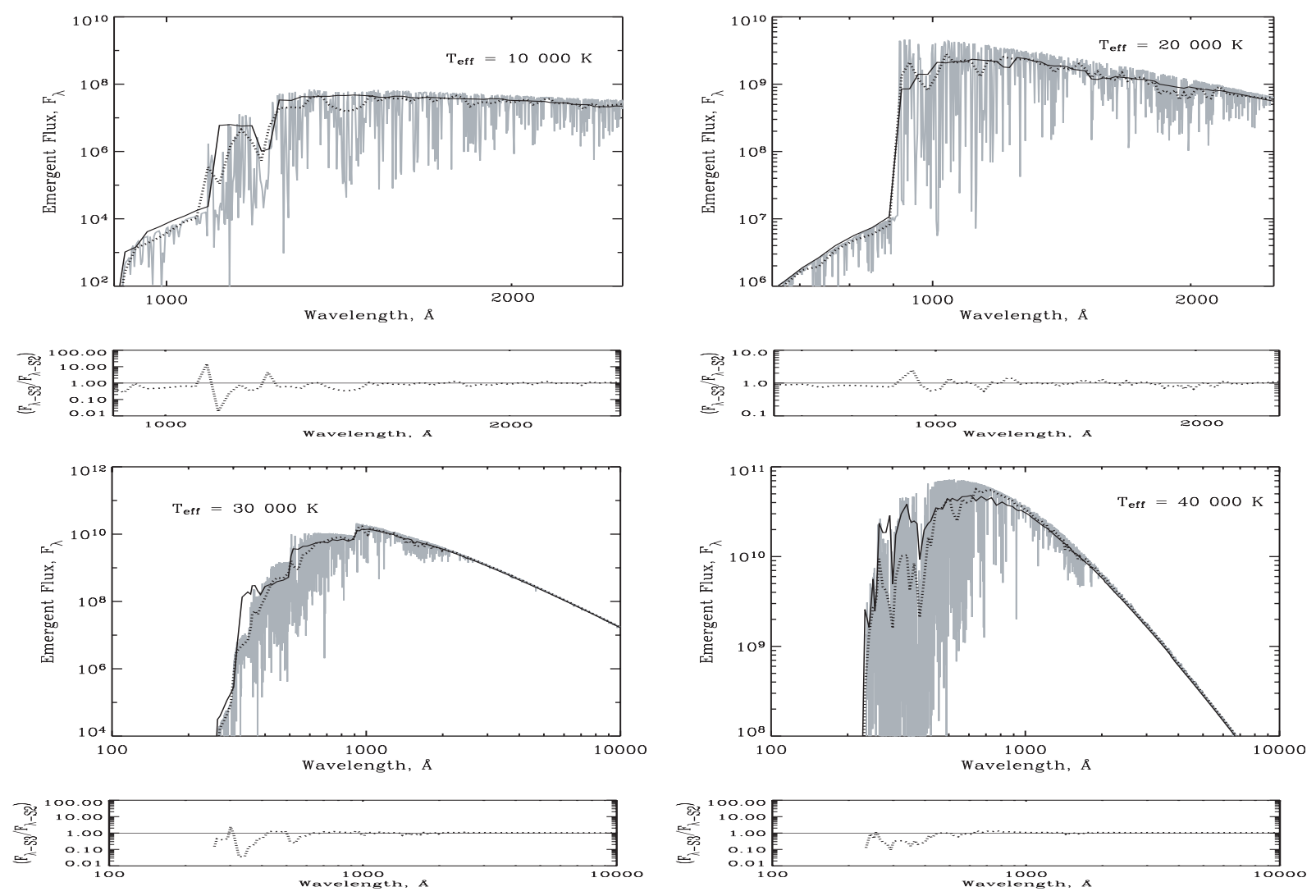

Fig. B.5. Flux distributions with varying effective temperatures, $\log g=3.00, n_{\mathrm{H}}=0.911, n_{\mathrm{He}}=0.089$ and solar metals for continuum plus line models. The STERNE2 model is shown by the solid line. The STERNE3 model is shown by the grey line, and is resampled to the STERNE2 model grid for comparison (dotted line).
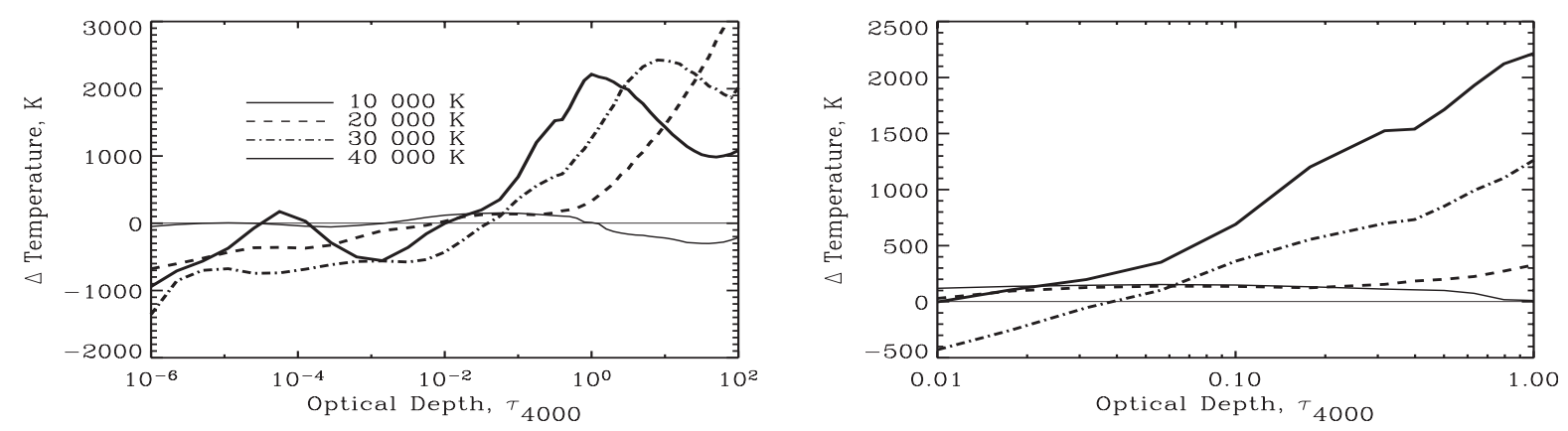

Fig. B.6. The difference in the temperature $\left(T_{\mathrm{STERNE} 3}-T_{\mathrm{STERNE} 2}\right)$ as a function of optical depth for the hydrogen-rich composition. The panel on the right shows the line forming region of the atmosphere. 
N. T. Behara and C. S. Jeffery: LTE model atmospheres with new opacities, Online Material p 6
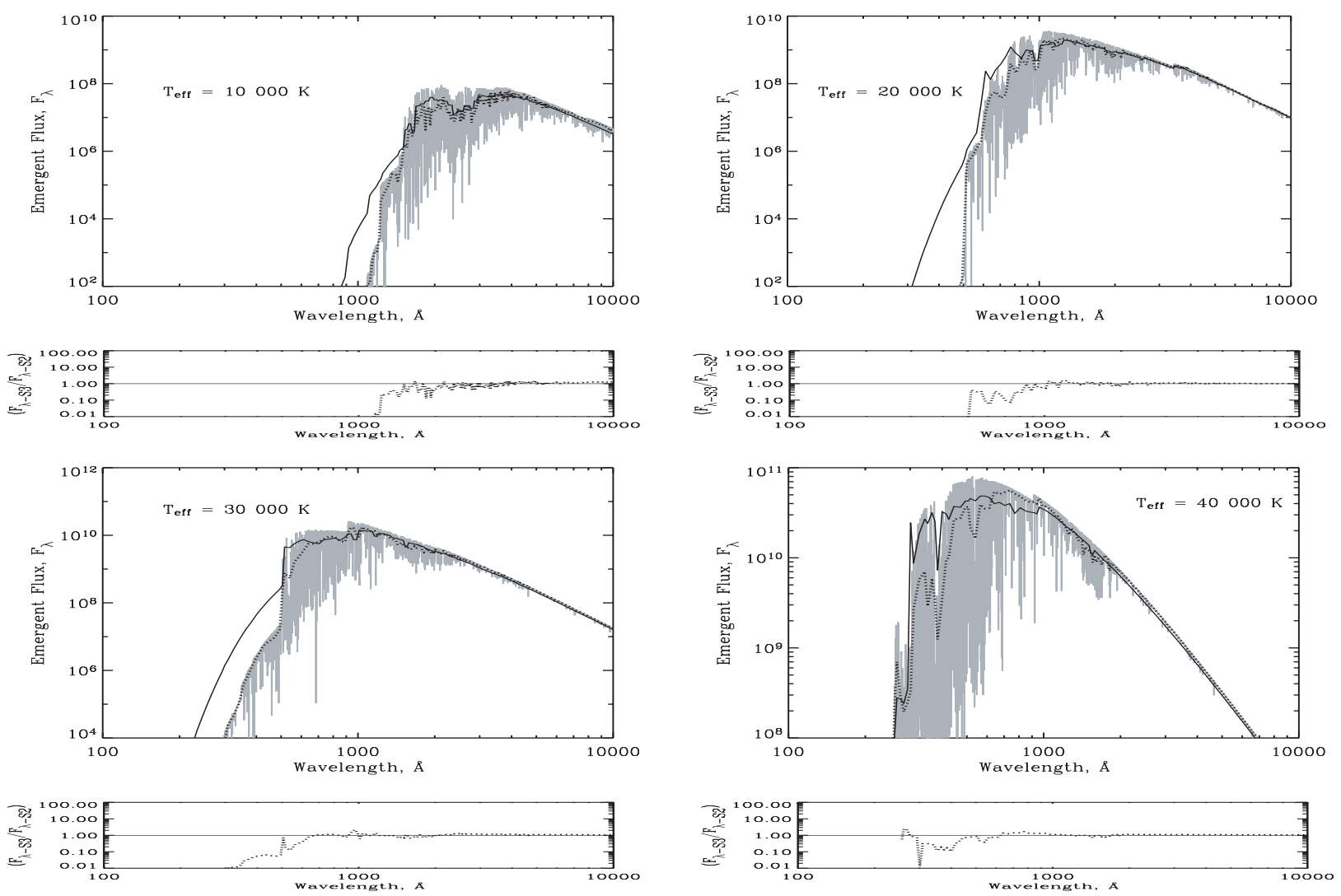

Fig. B.7. Flux distributions with varying effective temperatures, $\log g=3.00$, and composition $n_{\mathrm{H}}=0.0, n_{\mathrm{He}}=0.99$ and $n_{\mathrm{C}}=0.01$ for continuum plus line models. The STERNE2 model is shown by the solid line. The STERNE3 model is shown by the grey line, and is resampled to the STERNE2 model grid for comparison (dotted line).
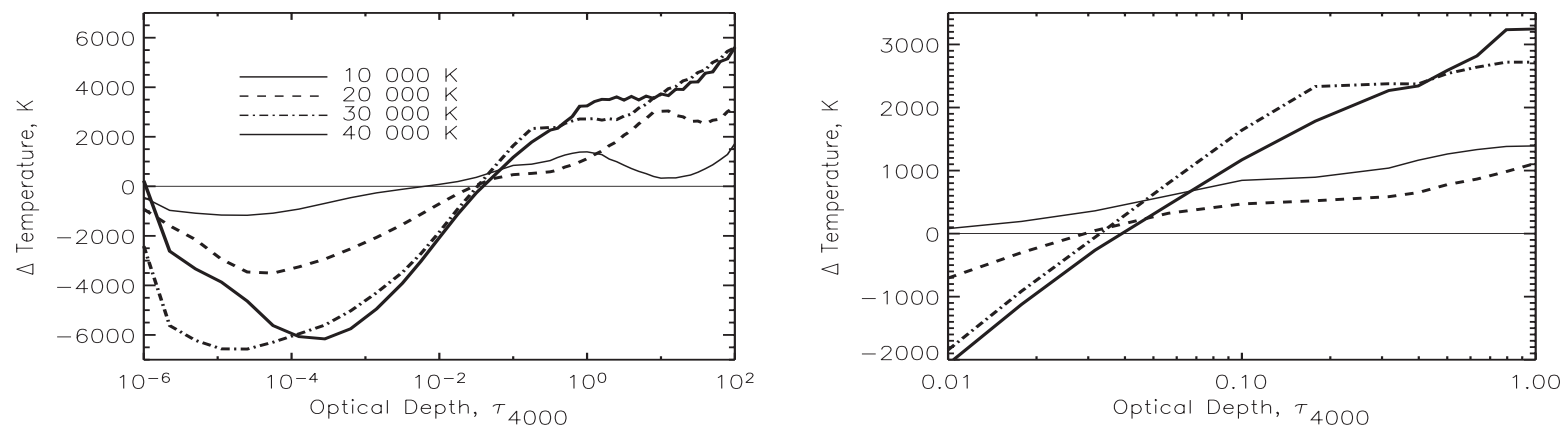

Fig. B.8. The difference in the temperature $\left(T_{\mathrm{STERNE} 3}-T_{\mathrm{STERNE} 2}\right)$ as a function of optical depth for the helium-rich composition. The panel on the right shows the line forming region of the atmosphere. 